\title{
Nonmonotonic settling of a sphere in a cornstarch suspension
}

\author{
Stefan von Kann, Jacco H. Snoeijer, Detlef Lohse, and Devaraj van der Meer \\ Physics of Fluids group, University of Twente, P.O. Box 217, NL-7500 AE Enschede, The Netherlands
}

(Received 4 August 2011; published 1 December 2011)

\begin{abstract}
Cornstarch suspensions exhibit remarkable behavior. Here, we present two unexpected observations for a sphere settling in such a suspension: In the bulk of the liquid the velocity of the sphere oscillates around a terminal value, without damping. Near the bottom the sphere comes to a full stop, but then accelerates again toward a second stop. This stop-go cycle is repeated several times before the object reaches the bottom. We show that common shear thickening or linear viscoelastic models cannot account for the observed phenomena, and propose a minimal jamming model to describe the behavior at the bottom.
\end{abstract}

DOI: 10.1103/PhysRevE.84.060401

PACS number(s): 82.70.Kj, 45.70.Vn, 47.50.-d, 47.57.ef

Concentrated particulate suspensions consist of a homogeneous fluid containing particles, larger than $1 \mu \mathrm{m}$. They can be found everywhere, and their flow is important in nature, industry, and even health care [1]. In spite of their significance, many aspects of the flow of these dense suspensions remain poorly understood. In order to study these materials, people have used methods inspired by classical rheology, and typically characterized them in terms of a constitutive relation of stress versus shear rate [2-6]. A general result is that, when increasing the shear rate, dense suspensions first tend to become less viscous (shear thinning) and subsequently shear thicken.

Probably the most conspicuous example of a dense suspension is formed by a high concentration of cornstarch in water. Recent rheological experiments in cornstarch have revealed the existence of a mesoscopic length scale [6,7], a shear thinning regime that terminates in a sudden shear thickening [8], a dynamic jamming point [4], and fracturing [9]. Merkt et al. [10] observed in a vertically shaken, thin layer of cornstarch suspension that, among other exotic phenomena, stable oscillating holes can be formed at certain frequencies and amplitudes [10,11], which were subsequently described using a phenomenological model based on a hysteretic constitutive equation [12]. At present, however, we are still far from a detailed understanding of dense suspensions.

In this Rapid Communication we subject a cornstarch suspension to a basic experiment, in which we observe and describe the settling of a spherical object in a deep bath of suspension. This yields two interesting observations. In the bulk, we find that the object velocity is oscillating in addition to going toward a terminal value. Near the bottom we observe a second phenomenon: The object comes to a full stop before the bottom, but then accelerates again, and this stop-go cycle can repeat up to seven times. We will show that both bulk and bottom behavior are conceptually different from that observed in a wide range of other fluids. We propose a jamming model for the stop-go cycles near the bottom that specifically includes the liquid-grain interactions.

Experiment. Our experimental setup is shown in Fig. 1(a). It consists of a $12 \times 12 \times 30 \mathrm{~cm}^{3}$ glass container containing a mixture of cornstarch and liquid. For the liquid we use either demineralized water or an aqueous solution of $\mathrm{CsCl}$ with a density of $1.5 \mathrm{~g} / \mathrm{cm}^{3}$, matching the density of the cornstarch particles. Experiments actually showed negligible differences between the density-matched and the unmatched liquid, except that for the unmatched liquid the suspension has to be stirred well prior to the experiment to counteract sedimentation. The cornstarch particles [Fig. 1(b)] are irregularly shaped and have a relatively flat size distribution of 5-20 $\mu \mathrm{m}$. Although we have varied the packing fraction $\phi$ of the cornstarch, for the data presented here we have fixed it to the high value of $\phi=0.44$, for which the phenomena of interest are particularly pronounced. All phenomena actually appear when $\phi \geqslant 0.38$. In a suspension of similarly sized spherical particles, we did not observe the phenomena reported here.

The settling sphere is a $d=4 \mathrm{~cm}$ diam pingpong ball, which is filled with copper beads to vary the buoyancy corrected mass $\mu=m_{\text {sphere }}-\rho_{S} \pi d^{3} / 6$ from 0 up to $137 \mathrm{~g}$. Here, $\rho_{S}$ is the density of the suspension. To measure the trajectory of the object inside the suspension, we follow tracers on a thin, rigid metal wire that is attached to the top of the ball (as in Ref. [13]) with a high-speed camera imaging at 5000 frames/s. From the trajectories the velocity and acceleration are determined at each time $t$ using a local quadratic fit around $t$ in a time interval of $12 \mathrm{~ms}$, corresponding to 60 measurement points.

In Fig. 2(a) we plot the time evolution of the velocity for three different, buoyancy-corrected masses $\mu$. For the smallest mass (green/light gray curve), after some transient directly following the impact (at $t=0$ ), there is an approximately exponential decay toward a terminal velocity, as would be found in a Newtonian liquid. When we increase $\mu$, we observe a much more abrupt decrease toward a terminal velocity, but in addition there are oscillations around this terminal value. This is seen most clearly for the highest mass in Fig. 2(a) (point 1). Second, instead of stopping at or very close to the bottom-as would happen in a Newtonian liquid — the object actually comes to a sudden, full stop (point 3) at $\sim 10 \mathrm{~mm}$ above the bottom for the highest $\mu$ [Fig. 2(a), inset]. ${ }^{1}$ Surprisingly, the object subsequently reaccelerates (4), only to come to another stop slightly closer to the bottom. This process is repeated several times until the bottom is reached. The observed phenomena are also present when we release the sphere from rest, but to avoid the long acceleration trajectory

\footnotetext{
${ }^{1}$ Careful examination of the data even reveals a very small negative velocity, corresponding to a tiny bounce upward, which can be interpreted as the elastic response of the jammed region of cornstarch underneath the sphere.
} 
(a)
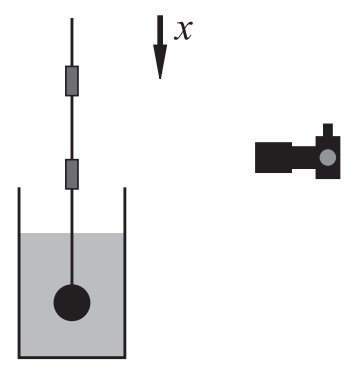

(b)

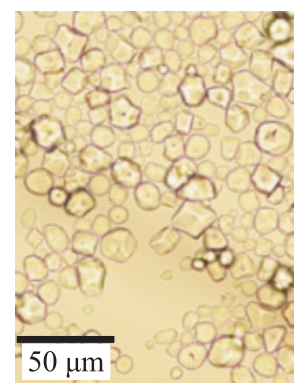

FIG. 1. (Color online) (a) Schematic view of the setup, consisting of the container filled with the suspension, the settling sphere with tracers attached, and a high-speed camera. For convenience, the positive direction of the vertical coordinate $x$ is chosen downward, with $x=0$ located at the bottom of the container. (b) Microscopic picture of the cornstarch grains.

for small $\mu$ we chose to impact the spheres with nonzero initial velocities to maximize the time in which the bulk effect is observable. To check that the bulk oscillations are not caused by interactions with the side walls, we changed the ratio of container to ball size, qualitatively leading to the same phenomena.

The motion of the settling sphere is described by

$$
m \ddot{x}=\mu g+D
$$
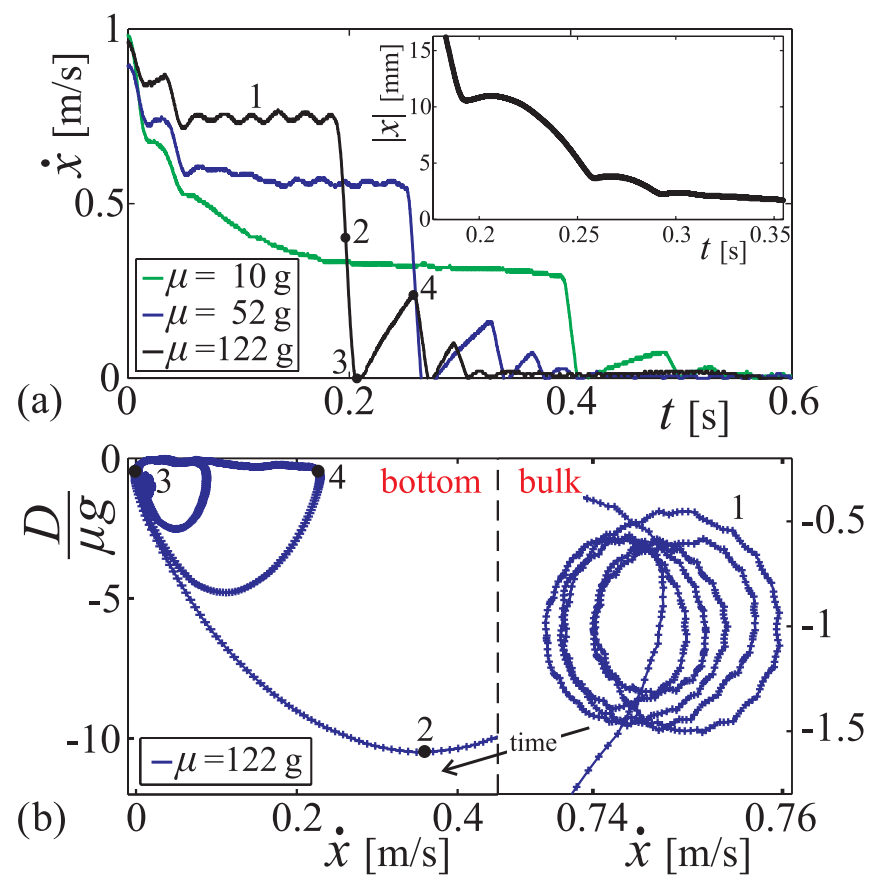

FIG. 2. (Color online) (a) Settling velocity $\dot{x}(t)$ of the settling sphere for three different masses $\mu=10,52$, and $122 \mathrm{~g}$. The inset shows the last part of the trajectory $|x(t)|$ for $\mu=122 \mathrm{~g}$. (b) Drag $D$ vs velocity $\dot{x}$ of the heaviest sphere in (a) $(\mu=122 \mathrm{~g})$. Note the different scales in the right and left half of the plot, which correspond to the bulk oscillations and the stop-go cycles at the bottom, respectively. In the latter, the drag force that causes the ball to come to an abrupt stop is up to ten times as large as gravity, and since it is limited by our fitting procedure, in reality it could be even higher. The numbers correspond to those in (a). where $D$ is the drag the sphere experiences inside the suspension and $m=m_{\text {sphere }}+m_{\text {added }}$ is the total inertial mass, including the added mass for which we will take the standard result $m_{\text {added }}=0.5 \rho_{S} \pi d^{3} / 6$. For a Newtonian fluid with a high dynamic viscosity $\eta$ we have $D=3 \pi \eta d \dot{x}$, leading to an exponential decay toward the terminal velocity $\dot{x}_{T}=$ $\mu g /(3 \pi \eta d)$. When we estimate the effective viscosity of our cornstarch suspension by identifying the (average) plateau velocities in Fig. 2(a), we find values between $\eta=0.87$ and $3.96 \mathrm{~Pa} \mathrm{~s}$, which are of the same order as found in Ref. [8], leading to Reynolds numbers on the order of $\mathrm{Re}=10$. This excludes that we are dealing with path instabilities associated with wake instabilities at $\operatorname{Re}>100$ in Newtonian fluids (see, e.g., Ref. [14]). In addition, we can also rule out a dominant influence from history forces arising from the buildup of the boundary layer for an accelerating object (e.g., the Basset force), as these are expected to be more pronounced for the lighter objects, in contrast to our observations.

We use Eq. (1) to determine the drag $D$ on the sphere as a function of its velocity [Fig. 2(b)]. From this plot it is clear that a given velocity in general corresponds to more than one value of the drag. Since non-Newtonian fluids with a monotonic stress-strain curve-as, e.g., power-law models for shear thickening and thinning fluids or yield stress fluid modelswill lead to a single-valued drag-velocity curve, we necessarily need to turn to a model that includes some history dependence.

Bulk oscillations. The behavior in the bulk is reminiscent of that of an object sinking in viscoelastic or stratified liquids for which oscillations are known to occur [15-17], albeit with two major experimental differences: First, for viscoelastic fluids there is an elastic rebound (oscillations in the position), whereas for our suspension the object continues to sink, with oscillations in the velocity. Second, in viscoelastic fluids the oscillation is observed to be strongly damped. From a modeling perspective, the damping term in linear viscoelastic fluid models accounts for both the decay of the oscillations and the approach of a terminal velocity. ${ }^{2}$ Clearly, such models fail to describe our observations: The terminal velocity is reached very rapidly after impact, while the oscillations persist without measurable damping.

Another approach is to consider a hysteretic model, such as the one proposed by Deegan [12] to explain why the "persistent holes" in vertically shaken cornstarch [10] do not collapse under hydrostatic pressure. We adapt this model by using a drag force $D$ in Eq. (1) which displays two states of damping with different effective viscosities: $D=-B_{1} \dot{x}$ when $|\dot{x}|$ falls below $\dot{x}_{1}$ and $D=-B_{2} \dot{x}$ when $|\dot{x}|$ rises above $\dot{x}_{2}$. Here, $B_{1}<$ $B_{2}$ and $\dot{x}_{1}<\dot{x}_{2}$, such that there exists a hysteresis loop. Such a model is capable of at least qualitatively describing any of our measurement series, with oscillations occurring when $\left|B_{1} \dot{x}\right|<\mu g<\left|B_{2} \dot{x}\right|$ : After impact, the object decelerates in the direction of a terminal velocity $\mu g / B_{2}$ until it reaches $\dot{x}_{1}$, after which a jump to the lower drag force branch occurs. Then it starts to accelerate toward a second terminal value $\mu g / B_{1}$, until $\dot{x}_{2}$ is reached and the system jumps back to the

\footnotetext{
${ }^{2}$ We have used the Maxwell model and variations thereof with one spring and up to two dashpots.
} 


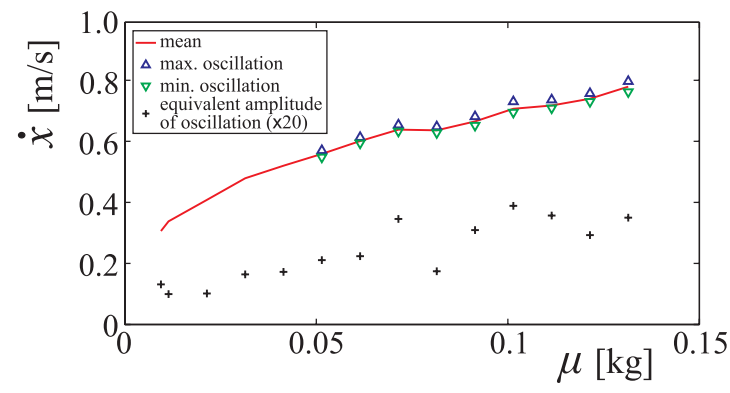

FIG. 3. (Color online) Bulk oscillations: Average (terminal) velocity, maximum and minimum velocity (when discernible), and equivalent amplitude of the oscillations, all as a function of the buoyancy-corrected sphere mass $\mu$. Oscillations are only discernible for $\mu>50 \mathrm{~g}$. Clearly, the minimum and maximum velocitieswhich should be identified with $\dot{x}_{1}$ and $\dot{x}_{2}$ in the model (see text), respectively—depend on $\mu$.

higher branch $\left(D=-B_{2} \dot{x}\right)$. This cycle repeats indefinitely, producing undamped oscillations all the way up to the bottom.

An important drawback of the model, however, is that the experimental findings can only be reproduced by adjusting $\dot{x}_{1}$ and $\dot{x}_{2}$ for every $\mu$. This can be appreciated from Fig. 3 , where we plot the average (terminal) velocity and the equivalent oscillation amplitude ${ }^{3}$ of the object in the bulk versus $\mu$. We see that both the terminal velocity, which should be identified with $\left(\dot{x}_{1}+\dot{x}_{2}\right) / 2$ in the model, and the equivalent amplitude $\left(\approx \dot{x}_{2}-\right.$ $\left.\dot{x}_{1}\right)$ increase with the buoyancy corrected mass. A similar trend was observed in Deegan's rheometer experiments [12]. This implies that the model for the drag force cannot be interpreted as a constitutive model for the cornstarch suspension, therewith greatly diminishing its predictive value.

Stop-go cycles at the bottom. Near the bottom we find a clear hysteresis between a situation with a sudden, violent deceleration [the large semicircular excursions of the drag force in the left-hand side of Fig. 2(b)] and a reacceleration period with a small, Stokes-like drag force $D$ [the almost horizontal parts in the same plot; also see the corresponding $\dot{x}(t)$ curves in Fig. 2(a)]. We interpret these stop-go cycles as follows: While the sphere is moving down, the cornstarch below it is slowly being compressed such that at a certain moment a jammed network of particles forms between the object and the container bottom. This jammed layer is responsible for the large force that brings the sphere to a full stop. Stresses build up in the network and therefore also within the interstitial fluid, which triggers a Darcy's flow in the porous medium formed by the cornstarch grains, allowing the network to relax through (small) particle rearrangements. This causes the jammed region to unjam and the object will start moving again. Such hardening of a cornstarch suspension has also been reported in Ref. [18], where a ball was pushed toward the bottom, leaving an indent on a clay layer on the bottom. This was attributed to forces being transmitted through a hardened layer beneath the ball.

\footnotetext{
${ }^{3}$ The equivalent oscillation amplitude is defined as $\sqrt{2}$ times the standard deviation of the velocity signal, which would be equal to the amplitude for a sinusoidal signal.
}

We model this process by coupling Eq. (1) to an equation for an order parameter which indicates whether or not the cornstarch suspension layer between the sphere and the bottom is jammed. We will take this to be the local particle volume fraction $\phi$. When $\phi$ exceeds a critical value $\phi_{\mathrm{cr}}$, the layer is jammed and the drag force $D$ is assumed to become infinitely large until the sphere comes to a standstill. This leads to the following modification of Eq. (1):

$$
\left\{\begin{array}{lll}
m \ddot{x}=\mu g+D & \text { when } & \phi<\phi_{\text {cr }} \\
\dot{x}=0 & \text { when } & \phi \geqslant \phi_{\text {cr }}
\end{array}\right\},
$$

with $D=-B \dot{x}$. The equation for the time rate of change of the packing fraction $\phi$ should contain a term that increases $\phi$ proportional to the compression rate $-\dot{x} / x$ of thecylindrical-layer of cornstarch below the sphere, which is the process by which the layer jams. Second, there should be a term that decreases $\phi$ through a relaxation process toward its equilibrium, bulk value $\phi_{\text {eq. }}$. This yields

$$
\dot{\phi}=-c \frac{\dot{x}}{x}-\kappa\left(\phi-\phi_{\mathrm{eq}}\right),
$$

in which $c$ and $\kappa$ are the proportionality constants of the compression and relaxation processes, respectively. Note that $\kappa^{-1}$ constitutes a time scale for the relaxation dynamics. The critical packing fraction $\phi_{\mathrm{cr}}$ is the value at which the cornstarch suspension dynamically jams. It must lie in between the

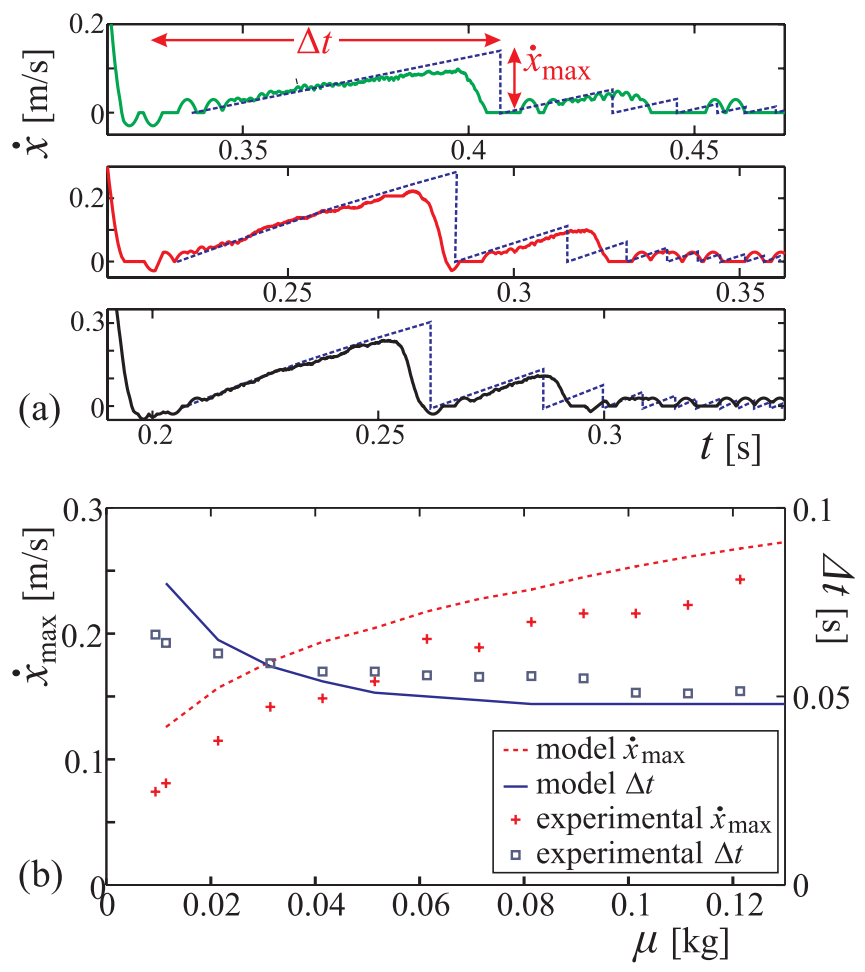

FIG. 4. (Color online) (a) Stop-go cycles: Comparison of the experimental velocity (solid lines) and that in the model (dashed blue lines) vs time for three different masses $(\mu=17,77$, and $132 \mathrm{~g}$ from top to bottom). Note that the time axis has the same scale in all three plots. (b) Stop-go cycles: Comparison of the reacceleration time $\Delta t$ (blue squares) and the maximum velocity $\dot{x}_{\max }$ (red crosses) reached after the first stop as a function of the buoyancy corrected mass $\mu$, for both the experiment (symbols) and model (lines). 
static, sedimented (0.44) and the maximally compacted $(0.57)$ value [19]. From creating the densest, still flowing cornstarch suspension in our laboratory, we estimate that $\phi_{\mathrm{cr}}=0.46$ at most. As a result, $\phi$ only varies marginally during the process, in agreement with recent research where during jamming of a cornstarch suspension in a Couette cell no density differences were measured within experimental accuracy (0.01) of the Magnetic Resonance Imaging (MRI) device used [8].

In Fig. 4(a) we compare the above model to our experiments for three different masses. We find that for a single value for $\kappa=40 \mathrm{~s}^{-1}$ and $c=0.025,{ }^{4}$ the model provides a reasonably good description of the stop-go cycles for all masses. Moreover, plotting the duration $\Delta t$ of a stop-go cycle and the maximum velocity $\dot{x}_{\text {max }}$ reached after the first stop yields the correct trend [Fig. 4(b)]. The fact that the second and higher stop-go cycles seem to be predicted too strong and fast by the model may be partly explained from the one dimensionality of the model, which does not fully describe the geometry of the settling sphere. Indeed, the model matches even better to preliminary experiments with a cylinder.

Finally, we connect the relaxation time scale $\kappa^{-1}$ from Eq. (3) to Darcy's law which, combined with continuity for an incompressible medium, leads to the porous medium equation $\partial \phi / \partial t=\left(k / \eta_{w}\right) \nabla^{2} \Delta P$ (see Ref. [20]). Here $\eta_{w}$ is the dynamic viscosity of water, $\Delta P$ the pressure, and $k$ the permeability, which is estimated using the Kozeny-Carman relation $k=$ $d_{g}^{2}(1-\phi)^{2} /\left(150 \phi^{2}\right)$, with $d_{g}$ the average grain diameter and $\phi \approx \phi_{\text {eq }}$. The left-hand side of the porous media equation is equal to the relaxation term in Eq. (3), i.e., $\kappa \Delta \phi$. The Laplacian

\footnotetext{
${ }^{4}$ The value $B$ we found near the bottom was fixed at $10 \mathrm{~kg} / \mathrm{s}$. The best fit for the parameters $B_{1}$ and $B_{2}$ lies at 5 and $15 \mathrm{~kg} / \mathrm{s}$ if we look at the experiment with the highest mass, thus in the same order of magnitude.
}

$\nabla^{2} \Delta P$ can be estimated as the ratio of the pressure generated in the packing due to the buoyancy-corrected weight of the sphere $\left[\Delta P \approx 4 \mu g /\left(\pi d^{2}\right)\right]$ divided by the square of the typical length scale $L$ over which the relaxation flow needs to take place to unjam the suspension. Taking the best-fit value $\kappa=40 \mathrm{~s}^{-1}$ and $\Delta \phi=\phi_{\mathrm{cr}}-\phi_{\mathrm{eq}}=0.02$ yields $L \approx 100 d_{g}$. This is of the same order as the mesoscopic length scale found by Bonnoit et al. [7], dominating the dynamics of highly concentrated cornstarch suspensions.

In conclusion, we presented experiments of objects settling into a bed of a cornstarch suspension, which revealed pronounced non-Newtonian behavior: Instead of reaching a terminal velocity and monotonously stopping at the bottom, the object's velocity oscillates within the bulk and goes through a series of stop-go cycles at the bottom. Common shear thickening and linear viscoelastic models fail to account for the observed phenomena, and we proposed a jamming model to describe the behavior at the bottom, which is in fair agreement with the experiment. A remaining question is to what extent a similar model would be able to explain the oscillations in the bulk. One could imagine that during the downward motion a layer of (nearly) jammed cornstarch forms around the sphere, as also proposed recently in Ref. [18], which somewhat increases drag and slows it down. This lower velocity, in turn, would allow the relaxation process to dissolve part of the jammed layer and the object would start to accelerate again. These competing effects would thus induce the oscillatory motion observed in the bulk. Clearly, more research is necessary to quantitatively substantiate such a mechanism.

The work is part of the research program of FOM, which is financially supported by NWO; S.v.K. acknowledges financial support.
[1] N. J. Wagner and J. F. Brady, Phys. Today 62(10), 27 (2009).

[2] H. A. Barnes, J. Rheol. 33, 329 (1989).

[3] A. Fall, N. Huang, F. Bertrand, G. Ovarlez, and D. Bonn, Phys. Rev. Lett. 100, 018301 (2008).

[4] E. Brown and H. M. Jaeger, Phys. Rev. Lett. 103, 086001 (2009).

[5] E. Brown, N. A. Forman, C. S. Orellana, H. Zhang, B. W. Maynor, D. E. Betts, J. M. DeSimone, and H. M. Jaeger, Nat. Mater. 9, 220 (2010).

[6] C. Bonnoit, T. Darnige, E. Clement, and A. Lindner, J. Rheol. 54, 65 (2010).

[7] C. Bonnoit, J. Lanuza, A. Lindner, and E. Clement, Phys. Rev. Lett. 105, 108302 (2010).

[8] A. Fall, F. Bertrand, G. Ovarlez, and D. Bonn (unpublished).

[9] E. E. Bischoff White, M. Chellamuthu, and J. P. Rothstein, Rheol. Acta 49, 119 (2010).

[10] F. S. Merkt, R. D. Deegan, D. I. Goldman, E. C. Rericha, and H. L. Swinney, Phys. Rev. Lett. 92, 184501 (2004).
[11] H. Ebata, S. Tatsumi, and M. Sano, Phys. Rev. E 79, 066308 (2009).

[12] R. D. Deegan, Phys. Rev. E 81, 036319 (2010).

[13] D. Lohse, R. Rauhé, R. Bergmann, and D. van der Meer, Nature (London) 432, 689 (2004).

[14] N. Mordant and J.-F. Pinton, Eur. Phys. J. B 18, 343 (2000).

[15] Benjamin Akers and Andrew Belmonte, J. Non-Newtonian Fluid Mech. 135, 97 (2006).

[16] Mark T. Arigo and Gareth H. McKinley, J. Rheol. 41, 103 (1997).

[17] N. Abaid, D. Adalsteinsson, A. Agyapong, and R. M. McLaughlin, Phys. Fluids 16, 5 (2004).

[18] B. Liu, M. Shelley, and J. Zhang, Phys. Rev. Lett. 105, 188301 (2010).

[19] J. L. Willett, Cereal Chem. 78, 64 (2001).

[20] D.-V. Anghel, M. Strauss, S. McNamara, E. G. Flekkoy, and K. J. Maloy, Phys. Rev. E 74, 029906(E) (2006). 